\title{
Progress in New Diagnosis and Therapeutic Strategy for Gastrointestinal Malignancy: Focus on New Molecular-Targeted Treatments
}

\author{
Toshiro Sugiyama \\ Department of Gastroenterology, Graduate School of Medicine and Pharmaceutical Sciences, University of Toyama, \\ Toyama, Japan
}

\section{Key Words}

Molecular-targeted treatment · Gastrointestinal stromal tumors · Neuroendocrine tumors - Japanese Gastroenterological Association

\begin{abstract}
The core symposiums of the Japanese Gastroenterological Association (JGA) annual scientific meetings focus on similar topics from year to year. The main topics of these symposiums for the last 3 years were centered on progress in new diagnostics and therapeutic strategies for gastrointestinal malignancy, with a special focus on new molecular-targeted treatments for gastrointestinal stromal tumors (GIST), neuroendocrine tumors (NET) and other gastrointestinal (GI) cancers, including malignant lymphoma, for which new molecular-targeted treatments are now being commonly used. The 8th annual meeting of the JGA was held in 2012 and 8 excellent papers were presented on progress in new diagnostics and therapy for GIST. The 9th annual meeting of the JGA was held in 2013 and 7 excellent papers were presented on new molecular-targeted treatments for colorectal carcinomas and Gl lymphoma. At the 10th annual meeting of the JGA, which was held in 2014, novel concepts of and therapeutic strategies for GI cancers, NET and GIST were discussed.
\end{abstract}

In 2010, the WHO proposed a new classification system in which NET was classified into three categories - NET-G1, NET-G2 and NEC - dependent on proliferative activity, and the term 'carcinoid' was deleted. Regarding GIST, several management guidelines have already been published: by NCCN in 2004, by ESMO in 2005, and in Japan in 2006. The Japanese guidelines have recently been revised. In addition to the summaries of the annual meetings from 2012 to 2014, the major points of the recently revised Japanese guidelines for the diagnosis and management of GIST are described in this review.

(c) 2015 S. Karger AG, Basel

\section{Introduction}

The core symposiums of the annual scientific meetings organized by the Japanese Gastroenterological Association (JGA) from 2012 to 2014 focused on similar topics. The main topics of these symposiums centered on new diagnostic progress and novel therapeutic strategies for gastrointestinal malignancies, with a special focus on new molecular-targeted treatments against neuroendocrine tumors (NET) [1] and gastrointestinal stromal tumors (GIST) [2-5]. Since the diagnosis of and novel therapeu- 
tic strategies for gastrointestinal malignancy have been widely investigated and are common issues in gastrointestinal (GI) tract medicine, many symposiums and workshops have been planned each year around these topics by each annual scientific committee. Therefore, in our core symposiums, we decided to focus on the specified fields of new molecular-targeted treatments for GIST and NET. The 8th annual meeting of the JGA was held in Sendai in February 2012, and was organized by Prof. Michio Hongo of Tohoku University, only 1 year after the suffering caused by the unprecedented earthquake in the Tohoku area. The 9th annual meeting of the JGA was held in Tokyo on the 25-26th of February, 2013, and was organized by Prof. Toshifumi Hibi of Keio University. The 10th annual meeting of the JGA was held in Fukushima on the 14-15th of February, 2014, and was organized by Prof. Seiichi Takenoshita of Fukushima Medical University. This paper will summarize these three core symposiums and will briefly review recent progress in these fields.

In 2010, WHO proposed a new classification system in which neuroendocrine tumors were classified into three categories, NETG-1, NET-G2 and NEC, depending on their proliferative activity, and the term 'carcinoid' was deleted [1]. Regarding GIST, several guidelines for their management were published by NCCN [2] in 2004, by ESMO [3] in 2005, and in Japan [4] in 2006. The Japanese guidelines have recently been revised [5], and these revisions are also discussed in this review.

\section{Summary of the 8th Annual Meeting}

At this annual meeting, 8 excellent papers were presented on new diagnostic procedures for and progress in the therapy of GIST. Nebiki et al. [unpubl. data] delivered a presentation regarding the usefulness of endoscopic ultrasonography (EUS) for preoperative diagnosis of gastric GIST. Eighty-nine cases were analyzed in this study and ultimately 65 cases were diagnosed as GIST and 23 cases as non-GIST tumors. Ulcer formation, the irregularity of the tumor margin, the presence of heterogeneous echo, high echo and nonecho findings within the tumor were compared between GIST and non-GIST. Only nonecho findings were significantly different between the two groups. However, the sensitivity of the nonecho findings for diagnosis of GIST before operation was $60 \%$ and this sensitivity was considered to be insufficient for diagnosis of GIST. Twenty cases of the 65 gastric GISTs underwent EUS-guided fine-needle aspiration (FNA) before surgery.
The diagnostic accuracy of EUS-FNA for gastric GIST was $100 \%$ and the MIB- 1 index determined by histological evaluation of EUS-FNA samples was consistent with that of surgical samples (90\%). Therefore, EUS-FNA was recommended for diagnosis of gastric GIST before surgery. Sugimori, Nakatani and colleagues [6] investigated how specific EUS findings might predict the growth of gastric submucosal tumors, because rapid growth is an important characteristic suggesting malignant GIST. Out of 46 cases in which EUS was conducted before and after GIST growth, $84 \%$ of those that showed low-echo findings in the first examination were growing during followup periods. Therefore, this EUS finding might be useful for predicting the growth of gastric GIST. Mitsui et al. [7] gave a presentation regarding the usefulness of doubleballoon endoscopy for the diagnosis of small intestinal GIST. Thirteen of 16 cases (81.3\%) showed ulcer formation on the tumors. Nine of 15 cases were diagnosed by histological examination; however, 4 cases showed bleeding after biopsy. Thus, although double-balloon endoscopy is very useful for diagnosis of GIST, the indication of biopsy for small intestinal GIST should be limited. Kataoka et al. [unpubl. data] presented a report regarding the usefulness of biopsy with endoscopic submucosal dissection (ESD) for the diagnosis of gastric GIST. Thirteen of 18 cases were diagnosed as GIST and 11 of these cases underwent surgery. The histological evaluations of 10 ESD biopsy samples from 11 cases (90.9\%) were consistent with those obtained by histologic evaluation of surgical samples regarding both the diagnosis and mitotic index. Therefore, biopsy with ESD is a safe and useful method for GIST diagnosis. Kataoka and Tanaka [8] presented their findings regarding the efficacy of photodynamic therapy using the photosensitizer, glycol-conjugated chlorin, which was taken up by the GIST cell line, GISTT1. In mouse models that were xenografted with GISTT1 cells that had taken up glycol-conjugated chlorin, irradiation of the tumor with light showed strong antitumor effects, mediated by induction of the apoptosis of GIST-T1 cells. Saito et al. [9] evaluated the long-term outcome of imatinib mesylate treatment of recurrent or unresectable GIST. Twenty patients were followed up for a median of 40 months (range 2.5-103) under imatinib mesylate treatment. The median progression-free survival (PFS) was 89 months and overall survival (OS) for 8 years was $67 \%$ (fig. 1). The B2222 trial is an up-front longterm follow-up study on the effectiveness of imatinib mesylate treatment against recurrent or unresectable GIST. This study reported that the PFS of patients treated with imatinib mesylate was around 60 months [10]. Thus, the 

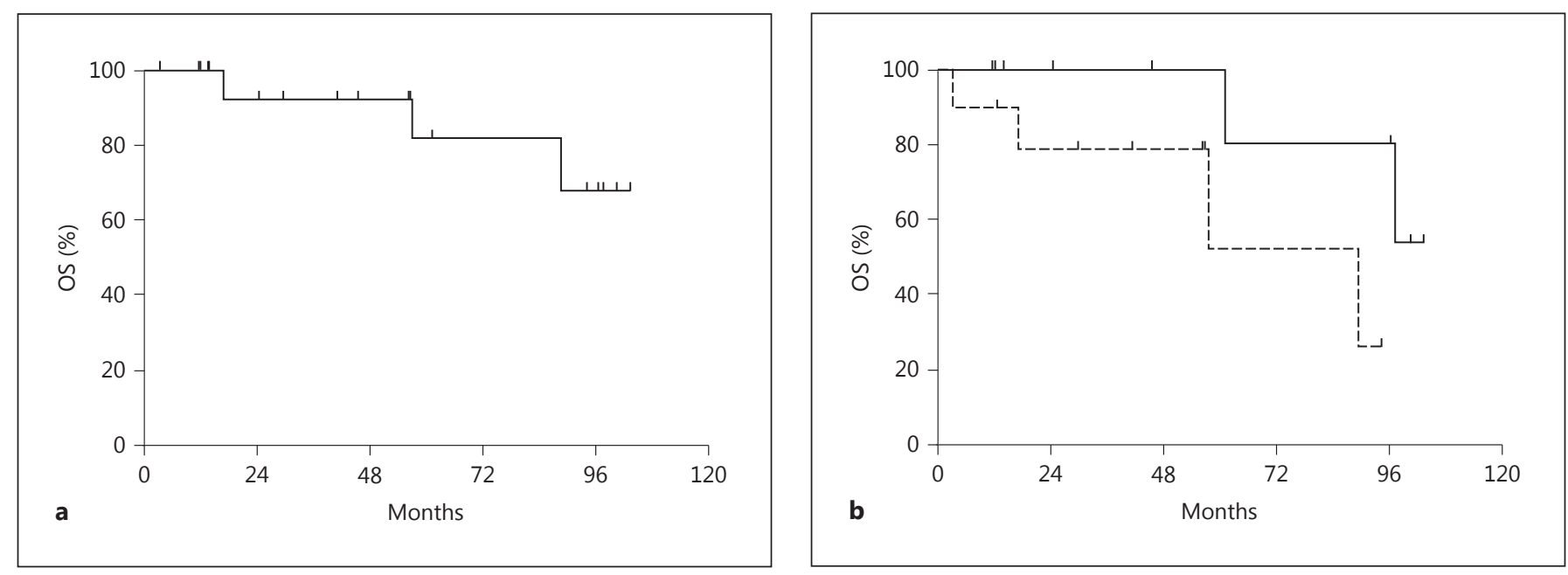

Fig. 1. a OS of metastatic/unresectable GIST treated with imatinib. b OS of imatinib-treated metastatic/unresectable gastric GIST (solid line) or nongastric GIST (dashed line). Modified from Saito et al. [9].

outcome of the study by Saito et al. [9], which was conducted in a single institute, was superior to that of the global trial, probably due to institute bias. Another interesting observation reported was that the size of the primary tumor prior to imatinib treatment may be one factor that predicts imatinib resistance. These results were published in Digestion in 2013 [9]. Yamamura et al. [unpubl. data] reported that the presence of the $c$-KIT exon 9 mutation in primary tumors, or hypertension or hypothyroidism as adverse events of sunitinib malate, were predictive factors for the effectiveness of sunitinib malate for imatinib-resistant GIST. Niinuma et al. [11] presented their findings that overexpression of miR-196a in GIST specimens is associated with high-risk grade, metastasis and poor survival. The miR-196a genes are located within the HOX gene clusters, and HOXC and HOTAIR genes were upregulated in GIST tissue. The group demonstrated that miR-196a and HOTAIR were useful biomarkers of and therapeutic targets for malignant GIST. These results were published in Cancer Research in 2012 [11].

\section{Summary of the 9th Annual Meeting}

At the 9th annual meeting, 7 excellent papers were presented regarding new molecular-targeted treatments against colorectal carcinomas and GI lymphoma. Yamada et al. [unpubl. data] presented their work regarding the detection of KRAS mutations in the circulating DNA of blood. They investigated KRAS mutations in surgical specimens and the corresponding circulating DNA of each of 5 patients with colorectal cancer. The KRAS mutations in 4 of the 5 circulating blood samples were the same as those in the corresponding surgical specimens of colon cancer tissues. In the other sample, KRAS mutations were not detected, probably due to the low tumor burden since these samples were derived from a patient with small pulmonary recurrence of colorectal cancer after surgical resection. Miyamoto, Okada and colleagues [12] gave a presentation regarding molecular imaging using the EGFR. The number of EGFRs on colorectal cancer cell lines was detected by flow cytometry after reaction with the EGFR monoclonal antibody, and the anticancer effect of anti-EGFR antibody was correlated with the presence of KRAS genotype, wild-type or mutant, which had already been reported in a human study. These results were confirmed in xenograft animal models using nude mice treated with anti-EGFR antibody, and were published in Digestion in 2014 [12]. Suzuki et al. [unpubl. data] presented a retrospective study regarding personalized treatment regimens for colorectal cancers, focusing on KRAS mutation, UGT1A1 polymorphism and combination with bevacizumab. When the effects of the chemotherapeutic regimens of FOLFIRI-3 $(n=54)$ were compared with those of FOLFIRI-3 plus bevacizumab ( $\mathrm{n}=$ 45), PFS was 14.0 and 20.7 months, respectively, and this difference was not statistically significant. However, OS of the FOLFIRI-3 group was 23.6 months, whereas that of the FOLFIRI-3 plus bevacizumab group was 40.4 months, and this difference was statistically significant. UGT1A1 
Fig. 2. Optimal morphologic response after treatment. a Baseline CT. b The first follow-up CT (optimal). Modified from Yoshita et al. [13].
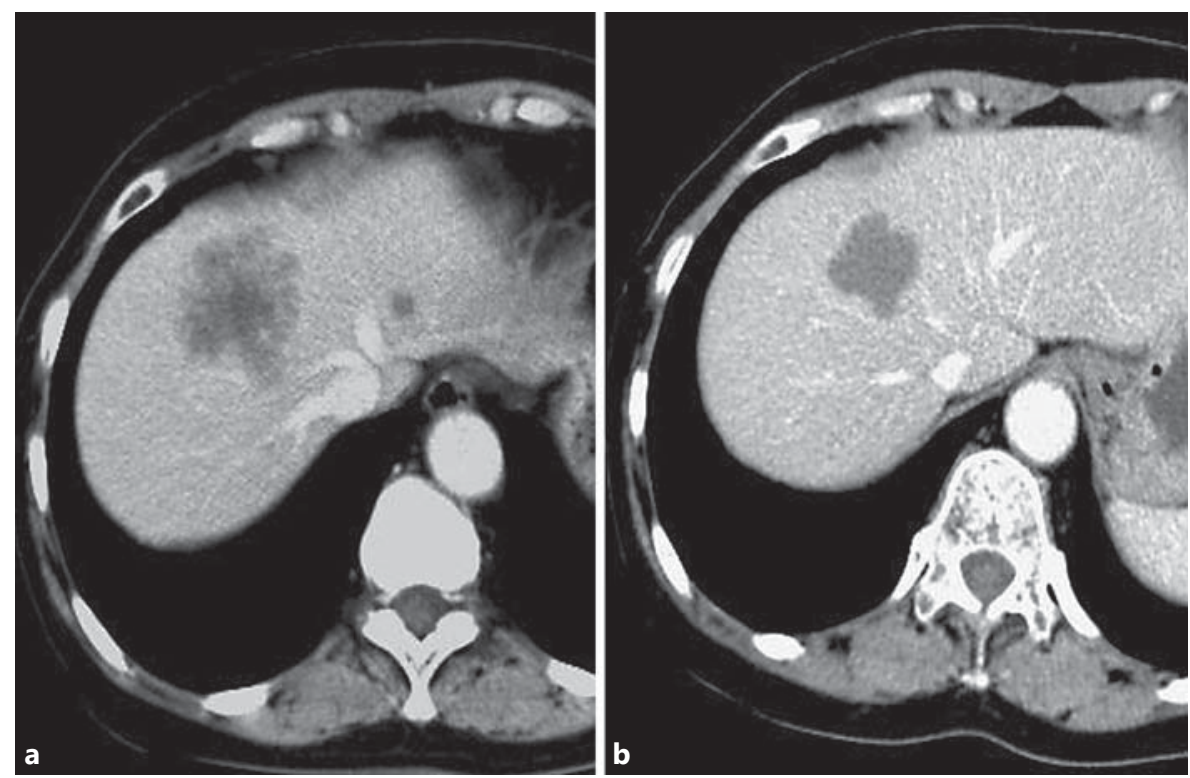

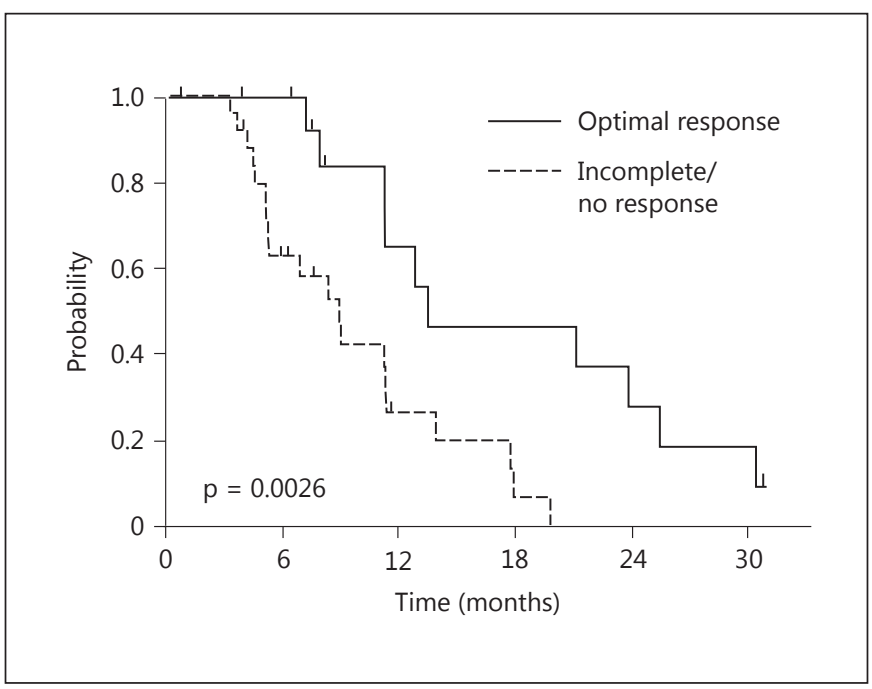

Fig. 3. PFS by morphologic response criteria. Modified from Yoshita et al. [13].

polymorphism was a prognostic factor for leukocytopenia due to the FOLFIRI-based regimen. Yoshita et al. [13] presented a new system for evaluation of the optimal morphologic response of colorectal cancer patients with liver metastasis to chemotherapy. RECIST is a standard system for the evaluation of solid tumors worldwide. However, because of recent new regimens, especially regimens including molecular-targeting drugs, development of a new system for optimal evaluation of solid tumors was proposed. The researchers assessed 41 colorectal cancer patients with liver metastasis. Three blinded radiologists evaluated computed tomography (CT) images after first-line fluorouracil-based chemotherapy with or without bevacizumab. Morphological response was assessed at the first follow-up CT (fig. 2). Optimal response was defined as homogenous morphology and hypodensity with a thin, sharply defined shape according to Chun et al. [14]. The PFS in the optimal-response group and the incomplete/no-response group is shown in figure 3 [13]. This new evaluation system will provide a good prognostic factor for the response of patients with liver metastasis to therapy. These results were published in Digestion in 2014 [13]. Ohshima et al. [unpubl. data] presented several biomarkers in tissues for evaluation of the prognosis of gastric or colorectal cancers. These biomarkers were determined by using tissue DNA microarray. Two hundred and two patients with colorectal cancers and $227 \mathrm{pa}-$ tients with gastric cancers who had survived for more than 5 years were investigated retrospectively. Ultimately, MT1-MMP, TIMP1, STC1, STC2, CCR7, Per2, IL24, REG4, PRL3 and IGFBP3 were identified as poor prognostic biomarkers of colorectal cancer and INHBA, SPARC and SULF1 were identified as poor prognostic biomarkers of gastric cancer. Nosho and colleagues [15] presented their research regarding whether the methylation of long interspersed element 1 (LINE-1) is related to the other molecular markers that are linked to the malig- 
nant potential of colorectal cancers. The average methylation level of LINE-1 was 53.0\% and LINE-1 extreme hypomethylation phenotype was correlated to tumor size, high clinical stage and the presence of distant metastasis of colorectal cancer. A prognostic impact of LINE-1 hypomethylation on the OS of patients with liver metastases was not observed. The concordance rate of LINE-1 extreme hypomethylation phenotype was $94 \%$ for KRAS, $100 \%$ for BRAF, $88 \%$ for mutated PIK3CA and $97 \%$ for microsatellite instability. Some of these results have already been published in the British Journal of Cancer [15]. Nakamura et al. [unpubl. data] presented the characteristics of follicular lymphoma in GI tracts in Japanese patients along with the effect of rituximab, an anti-CD20 monoclonal antibody. Thirty-six GI follicular lymphomas were examined. The entire GI tract, including the small intestine, was investigated. Twenty-four patients (67\%) showed lesions of nonlocalized follicular lymphoma in their GI tract. Four patients received surgical resection, 15 patients were treated with rituximab alone and 8 patients were treated with R-CHOP. Nine patients were under observation and awaited a treatment strategy. Although R-CHOP treatment was most likely to be chosen for stage IV lymphoma, PFS for 3 years was not different between the rituximab-alone group and the R-CHOPtreated group. Nonlocalized follicular lymphoma was a poor prognostic factor. Therefore, careful examination that includes the entire small intestine will be required for diagnosis and staging of GI follicular lymphoma.

\section{Summary of the 10th Annual Meeting}

The core symposium of the 10th annual meeting focused on a new concept and therapeutic strategy for GI NET [1] and GIST [2-5]. Since the pathological concept of these tumors is rapidly changing, two excellent overviews of this topic were presented by specialists in the field, Prof. Komatsu on GIST, and Prof. Sugihara on GI NET, and 4 papers were presented in the general session. Ogata et al. [16] presented their work regarding the localization of GIST ( $\mathrm{n}=234)$ and the effect of imatinib mesylate treatment on 41 recurrent or unresectable GISTs from 2001 to 2013. The primary organs were the stomach (72\%), the small intestine (15\%), the duodenum (3.8\%), the colon $(2.6 \%)$, the rectum $(0.9 \%)$ and the esophagus $(0.9 \%)$. Regarding the effect of imatinib mesylate, complete response was $17.1 \%$, partial response was $48.8 \%$, stable disease was $19.5 \%$ and the disease control rate was $85.4 \%$. OS over 5 years was $53.8 \%$ (median follow-up 4.0 years). Jiguchi et al. [unpubl. data] presented a series of 4 cases that were treated with imatinib mesylate and sunitinib malate. The first case was treated with surgical intervention and TAE intervention to a localized imatinibresistant lesion. In the second case imatinib mesylate treatment was ineffective, and there was a low serum concentration of imatinib mesylate due to polysurgery. The third case had two additional c-kit mutations and was treated with surgical intervention and sequential sunitinib malate. The fourth case had a PDGFRA D842V mutation. All 4 cases in this series provide support for the proposal of new therapeutic guidelines.

Ishii et al. [unpubl. data] presented a retrospective study of the pathological characteristics of 41 rectal NET. They concluded that the tumor size was an important factor predicting the progression of tumor as well as planning an effective treatment strategy. Finally, Nakamura et al. [unpubl. data] presented the expression of HER2/neu, VEGF, EGFR and SSTR in 36 colonic NET. HER2/neu displayed restricted expression in NEC (50\%) and was not expressed in NET-G1 or NET-G2. VEGF was expressed in $80 \%$ of NET-G1, $100 \%$ of NET-G2 and $50 \%$ of NEC. These findings suggest that these molecules will be new therapeutic candidates for NET.

\section{Updated Points of the Japanese Guidelines for the Diagnosis and Management of GIST}

\section{Diagnosis and Risk Classification}

Immunohistology of DOG-1 will be useful as an additional diagnostic method. DOG-1 shows restricted expression in GIST cells as well as in Cajal cells. Expression of DOG-1 has been reported to be similar to that of the KIT protein in several papers $[17,18]$.

The risk of recurrence depends on the tumor size, mitotic index, tumor rupture and origin. Since the frequency of gastric GIST is much higher in Japan than European countries, the Joensuu classification [19] is suitable for Japanese patients. In addition, to better understand the risk for patients, the figure indicating the risk is much more convenient. Therefore, the use of the modified Fletcher classification (Joensuu classification) and contour map [20] for identifying the risk of recurrence are recommended.

RECIST evaluation by CT scan is still a standard evaluation of tumor response to therapy. However, RECIST is not sensitive for evaluation of the effect of moleculartargeted treatments, especially in the early phase. The Choi criteria [21], which are new criteria for the evalua- 
tion of the effect of treatments, and FDG-PET evaluations are proposed for early and precise tumor evaluation.

\section{New Treatments}

The GRID trial [22] clearly demonstrated that regorafenib has higher efficacy and safety than the best supportive care in patients with imatinib and sunitinib failure. The median PFS of patients treated with regorafenib is 4.8 months, whereas that of patients treated with BSC is 0.9 months. Therefore, regorafenib is recommended as a third-line treatment for patients with imatinib and sunitinib failure or intolerance.

\section{Disclosure Statement}

No conflicts of interest exist for this publication.

\section{References}

1 Bosman ET, Carneiro F, Hruban RH, Theise ND (eds): WHO Classification of Tumours of the Digestive System, ed 4. Lyon, IARC press, 2010.

-2 Demetri GD, Benjamin R, Blanke CD, et al: NCCN Task Force report: optimal management of patients with gastrointestinal stromal tumor (GIST): expansion and update of NCCN clinical practice guidelines. J Natl Compr Cancer Netw 2004; (suppl 1):S1-S26.

-3 Blay JY, Bonvaltot S, Casali P, et al: Consensus meeting for the management of gastrointestinal stromal tumors: report of the GIST Consensus Conference of 20-21 March 2004, under the auspices of ESMO. Ann Oncol 2005; 16:566-578.

-4 Kubota T: Gastrointestinal stromal tumor (GIST) and imatinib. Int J Clin Oncol 2006; 11:184-189.

5 Japan Society of Oncology: GIST: gastrointestinal stromal tumor. http://www.jsco-cpg.jp/ item/03/index.html.

6 Nakatani M, Fujiwara Y, Sugimori S, et al: The usefulness of double-balloon enteroscopy in gastrointestinal stromal tumors of the small bowel with obscure gastrointestinal bleeding. Intern Med 2012;51:2675-2682.

7 Mitsui K, Tanaka S, Yamamoto H, et al: Role of double-balloon endoscopy in the diagnosis of small-bowel tumors: the first Japanese multicenter study. Gastrointest Endosoc 2009;70: 498-504.

8 Kataoka H, Tanaka M: PDT with a glucoseconjugated chlorin for GIST-response. Mol Cancer Ther 2014, Epub ahead of print.
-9 Saito S, Sugiyama T, Kajiura S, et al: Longterm follow-up outcome of imatinib mesylate treatment for recurrent and unresectable gastrointestinal stromal tumors. Digestion 2013; $87: 47-52$

10 von Mehren $\mathrm{MCH}$, Joensuu $\mathrm{H}$, Blanke CD, et al: Follow-up results after 9 years (yrs) of the ongoing, phase II B2222 trial of imatinib mesylate (IM) in patients (pts) with metastatic or unresectable KIT+ gastrointestinal stromal tumors (GIST). J Clin Oncol 2011; 29(suppl):abstr 10016.

11 Niinuma T, Suzuki H, Nojima, M, et al: Upregulation of miR-196a and HOTAIR drive malignant character in gastrointestinal stromal tumors. Cancer Res 2012;72:1126-1136.

12 Okada Y, Miyamoto H, Takayama T: Biomarkers for predicting the efficacy of antiepidermal growth factor receptor antibody in the treatment of colorectal cancer. Digestion 2014;89:18-23.

13 Yoshita H, Hosokawa A, Ueda A, et al: Predictive value of optimal morphologic response to first-line chemotherapy in patients with colorectal liver metastases. Digestion 2014;89: 43-48.

14 Chun YS, Vauthey JN, et al: Association of computed tomography morphologic criteria with pathologic response and survival in patients treated with bevacizumab for colorectal liver metastases. JAMA 2009;302:2338-2344.

15 Murata A, Baba Y, Watanabe M, Shigaki H, Miyake K, Ishimoto T, Iwatsuki M, Iwagami S, Sakamoto Y, Miyamoto Y, Yoshida N, Nosho K, Baba H: Methylation levels of LINE-1 in primary lesion and matched metastatic lesions of colorectal cancer. Br J Cancer 2013; 109:408-415.
16 Ogata K, Mochiki E, Ojima H, et al: A multicenter long-term study of imatinib treatment for Japanese patients with unresectable or recurrent gastrointestinal stromal tumors. J Surg Oncol 2014, Epub ahead of print.

17 West RB, Corless CL, Chen X, et al: The novel marker, DOG1, is expressed ubiquitously in gastrointestinal stromal tumors irrespective of KIT or PDGFRA mutation status. Am J Pathol 2004;165:107-113.

18 Miwa S, Sugiyama T, Murai Y, et al: Mutation assay of the novel gene DOG1 in gastrointestinal stromal tumors (GISTs). J Gastroenterol 2008;43:531-537.

19 Joensuu H: Risk stratification of patients diagnosed with gastrointestinal stromal tumor. Hum Pathol 2008;39:1411-1419.

20 Joensuu H, Vehtari A, et al: Risk of recurrence of gastrointestinal stromal tumour after surgery: an analysis of pooled population-based cohorts. Lancet Oncol 2012;13:265-274.

21 Choi H, Charnsangavej C, de Castro Faria S, et al: CT evaluation of the response of gastrointestinal stromal tumors after imatinib mesylate treatment: a quantitative analysis correlated with FDG PET findings. AJR Am J Roentgenol 2004;183:1619-1628.

22 Demetri GD, Reichardt P, Kang YK, et al: Efficacy and safety of regorafenib for advanced gastrointestinal stromal tumours after failure of imatinib and sunitinib (GRID): an international, multicentre, randomised, placebocontrolled, phase 3 trial. Lancet 2013;381: 295-302. 